\title{
Utilization of Malaria Diagnostic Tests and Receipt of Anti-Malarial Drugs by Febrile Patients Attending Outpatient Clinics of Health Centre IV Facilities in Mukono District, Uganda
}

\author{
Rose Naigino*, Achilles Katamba, Harriet M. Babikako, Aggrey Mukose \\ Makerere University School of Public Health College of Health Sciences, Kampala, Uganda \\ Email: rosenaigino@gmail.com, akatamba@yahoo.com, babikako@yahoo.com, amukose@musph.ac.ug
}

Received 3 May 2014; revised 15 June 2014; accepted 30 June 2014

Copyright (C) 2014 by authors and Scientific Research Publishing Inc. This work is licensed under the Creative Commons Attribution International License (CC BY). http://creativecommons.org/licenses/by/4.0/ cc) (i)

\section{Abstract}

Background: Failure to demonstrate the presence of malaria parasites prior to treatment with anti-malarial drugs remains a challenge in Uganda, often resulting into over-prescription of antimalarial drugs to febrile patients suspected of malaria. The aim of this study was to describe the role of utilization of malaria diagnostic tests and associated factors in the receipt of anti-malarial drugs among febrile patients suspected of malaria. Methods: In a cross-sectional study design, client-exit interviews with febrile patients and key-informant interviews with purposively selected health workers were conducted at outpatient clinics of health centre IV facilities in Mukono district. Data entry and analysis were done using EpiData 3.2 and STATA 10 respectively. Data were described using frequency distributions and proportions. Chi square was used in two by two tables, odds ratios as the measure of association and an alpha level of 0.05 was used in all significance tests. Results: Out of 408 respondents, the majority were female $(252,61.8 \%)$ and a third of the samples were aged five years and below. The mean age in years was 3.3 with a standard deviation of 2.1. More than half of the respondents $(359,88 \%)$ utilized malaria diagnostic tests and about half $(241,59 \%)$ received anti-malarial drugs. There were no statistically significant differences between utilizers and non-utilizers in most characteristics except age, history of indoor residual spraying and perceived satisfaction with services at outpatient clinics. Utilizers were $\mathbf{7 5 \%}$ less likely to receive anti-malarial drugs than non-utilizers after controlling for age, sex and residence (OR: 0.25, 95\%CI: 0.09, 0.66). Frequent power cut-offs as well as limited knowledge on malaria treatment guidelines amongst laboratory personnel were some of the major limitations to

\footnotetext{
${ }^{*}$ Corresponding author.
}

How to cite this paper: Naigino, R., Katamba, A., Babikako, H.M. and Mukose, A. (2014) Utilization of Malaria Diagnostic Tests and Receipt of Anti-Malarial Drugs by Febrile Patients Attending Outpatient Clinics of Health Centre IV Facilities in Mukono District, Uganda. Health, 6, 1628-1642. http://dx.doi.org/10.4236/health.2014.613195 
microscopic diagnosis of malaria. Conclusion: Utilizers were $75 \%$ less likely to receive anti-malarial drugs as opposed to non-utilizers. This implies that increasing utilization of malaria diagnostic tests can reduce the problem of over-prescription of anti-malarial drugs by $75 \%$ among those tested for malaria, since anti-malarial drugs would be received by only those with a parasitologically-confirmed diagnosis of malaria. Policy implications: To overcome the problem of overprescription of anti-malarial drugs, there must be a policy that ensures a consistent power supply in all public health laboratories. Training of health workers should encompass all cadres and work-shifts for laboratory personnel should be established to enhance utilization of malaria diagnostic tests especially at night.

\section{Keywords}

\section{Malaria Diagnostic Test, Febrile, Malaria Suspect, Utilizers, Receipt of Anti-Malarial Drugs}

\section{Introduction}

Worldwide, malaria caused an estimated 3.4 billion clinical cases and 627,000 deaths in 2012, 80\% $(562,000)$ of which occurred in tropical Africa where the majority of deaths $(82 \%, 462,000)$ occurred in children below five years of age [1].

In 2012, malaria accounted for an estimated 1500 admissions and 20 deaths per 100,000 population in Uganda. According to the World Malaria report 2013, the prevalence of malaria in Uganda was presented at $90 \%$ in the high transmission areas ( $>1$ case per 1000 population) and 10\% in the low transmission areas ( 0 - 1 cases per 1000 population) [1].

In Mukono district, findings from a house-hold survey done to assess self-reported malaria showed that approximately $52 \%$ of the 5583 households surveyed reported at least a person with malaria two weeks prior to the survey date [2]. In a report from Mukono district health office, malaria is rated number one (39\%) among the top five causes of morbidity for all age-groups, followed by pneumonia (9\%), intestinal worms (6\%), oral and skin diseases (4\%) [3].

Uganda's health care system currently consists of Village Health Teams (VHTs), health centre II, III and IV facilities, District Hospitals, Regional and National Referral Hospitals (NRH) [4].

Health centre I facilities have no structural establishment. These are composed of village members who deal with health-related activities such as drug distribution and health education. Health centre II facilities serve a parish and are headed by a nurse. These provide basic health services to the parish, including out-patient care, antenatal care and immunization. Health centre III facilities serve a sub-county and are headed by a medical/clinical officer. They provide all health services of health centre II facilities plus in-patient care and environmental health. Health centre IV facilities serve a health sub-district. They offer all services of health centre III facilities plus minor surgeries, including supervision of all activities in health centre II and III facilities in that sub-district. District hospitals offer both in-patient and out-patient care while regional and national referral hospitals offer specialized services in addition to general services [4].

In Mukono district, the syndromic approach (treatment based on clinical symptoms) is the commonest method of malaria diagnosis practiced by health workers, thereby increasing the likelihood that some patients will receive anti-malarial drugs irrationally [5].

Although findings presented in the World Malaria report 2013 showed an increase in the utilization levels of malaria diagnostic tests for suspected cases of malaria of all age groups in Uganda from 20 to 75 parasitologically-confirmed cases per 1000 between 2002 to 2012, [1] utilization of malaria diagnostic tests by febrile patients of all age-groups suspected of malaria at outpatient clinics of health centre IV facilities in Mukono district was estimated at 57\% in 2010/2011. This proportion (57\%) was low compared to the national requirements for effective malaria case management in Uganda, which entails 100\% parasitologically-confirmed diagnosis for all suspected cases of malaria. According to a report from Mukono district health office, out of 18,170 febrile patients registered in all the three health centre IV facilities in 2010/2011, an estimated 53\% (9665) tested positive for malaria and 11,315 including an excess of 1650 (15\%) febrile patients (who did not have a parasitologically- 
confirmed diagnosis of malaria) received ACT in the same year [3]. This implies that almost $15 \%$ of the febrile patients who received artemisinin-based combination therapy did not have a parasitologically-confirmed diagnosis of malaria, but an indication that there may be over-prescription of ACT, often resulting in frequent drug stock-outs. Without malaria confirmation, it is difficult to rule out non-malaria fevers. The true burden of the disease becomes difficult to consequently quantify the drug resistance and wastage of anti-malarial drugs on unnecessary treatment [6] [7].

The aim of this study was to describe patients' and facility-based factors associated with utilization of malaria diagnostic tests among febrile patients suspected of malaria at outpatient clinics of health centre IV facilities in Mukono district between March and May 2012. This study was also done to describe provider-related factors associated with utilization of malaria diagnostic tests for out-patients in health centre IV facilities in Mukono district, between March and May 2012. In addition, the study aimed at determining whether there is an association between utilization of malaria diagnostic tests and receipt of anti-malarial drugs amongst febrile patients suspected of malaria at out-patient clinics of health centre IV facilities in Mukono district, so as to provide a broader understanding of the role of utilization of malaria diagnostic tests in the receipt of anti-malarial drugs among febrile patients suspected of malaria.

\section{Methods}

\subsection{Study Area}

The study was conducted in Mukono district (Uganda), which borders the districts of Jinja in the East, Kayunga in the North, Luwero in the West and Kalangala in the South. According to the 2002 national census figures, Mukono district had a population of about 807,900 , almost $49 \%$ of whom were male and $50.2 \%$ were female [8]. Mukono district has a total population of 42 Government-aided public health facilities including three health centre IV facilities, 14 health centre III facilities and 25 health centre II facilities. This study was exclusively implemented at all the three health centre IV facilities found in Mukono district which included: Mukono health centre IV, Kojja health centre IV and Mukono Church of Uganda (C.o.U) health centre IV. On average, each health centre IV facility is served by two medical doctors, four nursing officers, three clinical officers and two laboratory technicians.

Mukono district was chosen as study area because of the high malaria prevalence (51.9\%) in this district [2]. The study was exclusively implemented in health centre IV facilities because the Uganda National Diagnostic Policy permits use of the blood smear microscopic method of malaria diagnosis only in health centre IV facilities while RDTs are encouraged in lower level health facilities such as health centre III and II where there's limited power supply [3].

\subsection{Study Design}

It was a cross-sectional study involving client-exit interviews with febrile patients suspected of malaria at outpatient clinics of Mukono health centre IV, Kojja health centre IV and Mukono C.o.U health centre IV in the period March and May 2012. The study population was composed of febrile patients of all age-groups suspected of malaria at outpatient clinics of all the three health centre IV facilities in Mukono district between March and May 2012. Additionally, key informant interviews were conducted with purposively selected health workers to provide an understanding of our quantitative findings.

\subsection{Eligibility Screening}

Eligibility screening of potential study participants was done on the basis of patients' presenting symptoms, clinician's suspected diagnosis on presenting patient, patients' referral for malaria diagnosis and patients' history of malaria diagnosis for the same malaria episode. Patients whose cards read at least a word written "rule out malaria" were ultimately eligible for the study while those whose cards did not have any "malaria" word written anywhere were excluded from the study as this implied that malaria was not one of the probable illnesses initially suspected by the clinician. All eligible participants who consented to participate were given participant cards with identification numbers to identify them as study participants at the different interview points while those who were too sick to respond were excluded. 


\subsection{Sample Size and Sampling Method}

The sample size for client-exit interviews was estimated using the Kish-Leslie's formula for cross-sectional studies with a 6\% non-response rate [9] resulting into a sample size of 408.

Details of the sample size calculation done are presented below:

$$
N=\frac{p(1-p) Z^{2}}{d^{2}}=N=\frac{0.5 \times 0.5 \times 1.96^{2}}{0.05^{2}}=385(6 \% \text { non-response rate })=408
$$

where; $N=$ number of respondents needed, $p=$ estimated proportion of febrile patients who receive care at health centre IV facilities in Mukono district.

In this study, the sample size of 408 respondents was obtained under the following assumptions:

A p $=50 \%$ prevalence was used to mean at least $50 \%$ of febrile patients at outpatient clinics of health centre IV facilities in Mukono district were tested for malaria. $Z$ was 1.96 (Z-score corresponding to 95\% confidence interval), $d$ = estimated error $=0.05$ and a $6 \%$ non-response rate was used based on a study conducted at outpatient clinics in Mulago (Uganda) in which the authors reported a 6\% non-response rate for exit interviews among clients who refused to participate because they did not have time to participate in the interviews [9].

The sampling method used was probability proportionate to size because the outpatient clinics of all the three health centres included in the study registered varying proportions of febrile patients per year with the majority recorded at Mukono health centre IV and the least at Mukono C.o.U health centre IV. Out of the 408 respondents required for client-exit interviews, the biggest proportion $204(50 \% \times 408)$ was drawn from Mukono health centre IV, $122(30 \% \times 408)$ from Kojja health centre IV and the least $82(20 \% \times 408)$ from Mukono C.o.U health centre IV. However, purposive sampling was used to select health workers for the key informant interviews.

\subsection{Definitions}

For the purpose of this study, febrile patients were defined as patients presenting with a history of fever with or without symptoms suggestive of malaria such as: headache, chills, vomiting and joint pains. A malaria suspect was defined as a febrile patient suspected of malaria whose patient card read a word "rule out malaria". Utilization of malaria diagnostic tests was measured from the patients' self reports of having obtained a blood test by finger prick, heel or vein puncture and given their test results from the laboratory. "Receipt of anti-malarial drugs was measured from the patients' self reports of having received anti-malarial drugs (such as: artemisinin-based combination therapy, sulphadoxine-pyrimethamine (also known as fansidar), quinine or any other type of anti-malarial drug) following prescription with or without prior diagnosis of malaria but also confirmed by reading the patient's card to check whether the anti-malarial drugs received had been initially prescribed by the clinician. Anti-malarial drugs were defined "available” if patients reported having received them from the health facility and "unavailable” if they reported having been referred to an external source such as a drug shop to buy anti-malarial drugs.

\subsection{Instruments}

The data collection tools used in this study included: assent and consent forms for study participants below and above 18 years of age respectively, an eligibility screening form, a semi-structured interviewer-administered questionnaire for client-exit interviews, an observational checklist to assess facility-based factors and a key-informant guide to capture data on provider-related factors. The key-informant interviews were conducted with a total of six purposively selected health workers who included three clinical officers and three laboratory technicians perceived to be more knowledgeable on this research subject. The questionnaires were translated from English to Luganda (the most commonly spoken local language in the study area) and back-translated by two English and Luganda speaking experts who worked independently from the Institute of Languages, Makerere University.

\subsection{Procedure and Study Variables}

\subsubsection{Specific Objective 1}

Quantitative data were obtained through client-exit interviews using a pre-determined semi-structured inter- 
viewer-administered questionnaire which consisted of a total of 19 question items. An observation checklist was used to collect data on facility-based factors which involved an assessment of the presence of utilities, supplies and equipment for microscopic diagnosis of malaria.

\subsubsection{Specific Objective 2}

Qualitative data on provider-related factors were obtained through key-informant interviews with a total of six purposively selected health workers using a key-informant guide that consisted of questions designed to assess and document their knowledge and training on malaria treatment guidelines, basis for referring malaria suspects for malaria diagnosis as well as their prescription practices for malaria suspects (Table 1).

\subsubsection{Specific Objective 3}

Utilization of malaria diagnostic tests (the main independent variable) was measured from the patients' self reports of having received a blood test by finger prick, heel or vein puncture and given their laboratory test results while receipt of anti-malarial drugs (the main outcome variable) was measured from the patients' self report of having received anti-malarial drugs following prescription by the health worker.

\subsection{Quality Control}

Patients' cards were read to verify the type of anti-malarial drugs prescribed against the national recommended drug list for malaria treatment. The tools were pretested with ten respondents at Kawempe health centre IV to ascertain whether the question items were correctly understood and interpreted in the same way by both the interviewers and respondents. After the pretest, the tools were modified where necessary to improve on the clarity and understandability of the question items. Research assistants were trained and closely supervised in the field to ensure quality and consistency of the data collected.

Table 1. Summary of data sources and variable by objective.

\begin{tabular}{ll}
\hline Objective & Type of data \\
\hline Specific objective 1 & Quantitative data \\
To describe patients' and facility-based factors & -Client exit interviews \\
associated with utilization of malaria diagnostic tests & with febrile patients. \\
among febrile patients suspected of malaria at & -Checklist to assess \\
out-patient clinics of health centre IV facilities in & facility-based factors.
\end{tabular}

Study variables

Independent variables

\section{Patient factors}

-Age, sex, education level, occupation.

-Marital status, visiting status, type of residence,

pregnancy status for females aged 15 - 49, history of sickle cell disease diagnosis, HIV status.

-History of anti-malarial drug use on current malaria episode, history of bed net use, history of indoor

residual spraying, perceived overall satisfaction with services.

\section{Facility-based factors}

-Presence of utilities, supplies, equipment required

for microscopic diagnosis of malaria.

-Number of skilled laboratory personnel.

-Number of prepared blood smears per clinic day, availability

of anti-malarial drugs.

\section{Outcome variable}

Utilization of malaria diagnostic tests by febrile patients suspected of malaria.

\section{Specific objective 2}

To describe provider-related factors associated with utilization of malaria diagnostic tests at out-patient clinics of health center IV facilities in Mukono district between March and May 2012.

\section{Specific objective 3}

To determine whether there is an association between utilization of malaria diagnostic tests and receipt of anti-malarial drugs among febrile patients suspected of malaria at out-patient clinics of health center IV facilities in Mukono district between March and May 2012.

\section{Qualitative data}

-Key informant interviews with six purposively selected health workers.

Quantitative data -Client exit interviews with febrile patients.

\section{Themes}

-Cadre of health worker and training. -Knowledge of malaria treatment guidelines.. -Basis for referral of malaria suspects for malaria diagnosis. -Prescription practices for malaria suspects.

Main independent variable

Utilization of malaria diagnostic tests by febrile patients suspected of malaria.

\section{Outcome variable}

Receipt of anti-malarial drugs. 


\subsection{Data Management and Analysis per Specific Objective}

\section{1) Objective 1}

A data-entry sheet was prepared using EpiData version 3.02 software and all variables coded with reference to their respective question items in the questionnaire. For continuous variables such as age, individual responses were directly entered and codes were used for categorical variables. Data cleaning was done and the cleaned data exported to STATA version 10.0 for subsequent analysis. For this objective, the unit of analysis was the individual study participant and the health facility. To begin with, univariable analysis for each independent factor was done to understand the distribution of each factor in terms of frequencies and proportions while means and standard deviations were obtained for continuous variables such as: age. Frequency distributions and proportions were used to describe the sample population. Bivariable analysis then followed to understand the distribution of each independent factor across the outcome of utilization of malaria diagnostic tests. A new binary outcome variable for 'utilization of malaria diagnostic tests' which consisted of two categories (that is: utilizers and nonutilizers of malaria diagnostic tests) was generated. Chi square was used in two-by-two tables to identify statistically significant differences between utilizers and non-utilizers of malaria diagnostic tests across their sociodemographic and behavioural characteristics such age, sex, time of arrival at the health facility, among other factors. Utilizers and non-utilizers of malaria diagnostic tests were considered statistically different across a given characteristic if the chi square p-value obtained was less than 0.05 . Results for this objective were presented in tabular form while bar graphs were used to present findings on facility-based factors.

\section{2) Objective 2}

Narrative data from each key-informant was transcribed verbatim and read repeatedly to check the content for completeness of responses and quality of transcripts. Data were organized by question under the different themes relating to the four variables on provider-related factors namely; cadre of health worker and training, their knowledge on malaria treatment guidelines, basis for referral of suspects for malaria diagnosis and prescription practices for malaria suspects (Table 1). The purpose of this approach was to look across all respondents and their answers to be able to identify consistencies and differences by tallying similar responses. These data were then analyzed manually using an Excel sheet and typical quotes from the key informants were presented verbatim in the results [10].

\section{3) Objective 3}

To begin with, bivariable analysis was done to identify independent variables that showed an association with the outcome variable of "receipt of anti-malarial drugs". At bivariable analysis, all associations were considered statistically significant at a $\mathrm{p}<0.05$. Multivariable analysis using logistic regression was done to examine the association between "utilization of malaria diagnostic tests" and "receipt of anti-malarial drugs" while controlling for multiple predictor variables in the model. Logical model building was done by systematically introducing one variable stepwise at a time into the model. Inclusion of variables in the adjusted model was done on the basis of factors that had a p-value less than 0.05 at bivariable analysis or empirically known potential confounders from known scientific literature. Dummy variables were generated to allow analysis across subcategories of each independent factor on "receipt of anti-malarial drugs". Odds ratios were used as the measure of association and an alpha level of 0.05 was used in all significance tests. The final model was tested for goodness of fit using Lfit goodness of fit test with smaller p-values $(\mathrm{p}<0.05)$ indicating poor fit. The results for this objective were presented in tabular form.

\subsection{Study Limitations}

Interviewer bias was a potential study limitation which was overcome by training and continuous supervision of research assistants in the field during data collection.

\section{Ethical Consideration}

The study protocol was approved by Makerere University School of Public Health-Institutional Review Board. Written informed consent and assent were obtained from all potential study participants aged above and below 18 years respectively. Confidentiality was maintained through the use of codes and participant identification numbers on the questionnaires other than names so as to mask the identity of individual study participants. Permission to conduct the study was obtained from Mukono district health office and management of the respective health facilities. 


\section{Results}

Out of 472 potential study participants screened for eligibility, 48 were not eligible and 16 out of the 424 eligible participants refused to participate, resulting in an overall response rate of $(408 / 424 \times 100)=96.2 \%($ see Figure 1).

\subsection{Description of the Sample Population}

Out of 408 respondents, the majority $(120,29.4 \%)$ were children aged below five years and a third of the sample were female (252, 61.8\%). The mean age in years of the respondents was 3.3 with a standard deviation of 2.1. A greater proportion of the adult population was not employed (73.8\%) and about half the sample (216, 52.9\%) lived in the urban parts of Mukono district (Table 2).

Table 2. Background characteristics of the 408 febrile patients suspected of malaria at outpatient clinics of health centre IV facilities in Mukono district between March-May 2012.

\begin{tabular}{|c|c|c|c|}
\hline Characteristic & Sub-categories & Frequency(n) & Percentage (\%) \\
\hline \multirow[t]{9}{*}{ Age-group } & $0-4$ & 120 & 29.4 \\
\hline & $5-10$ & 69 & 16.9 \\
\hline & $11-17$ & 34 & 8.3 \\
\hline & $18-27$ & 74 & 18.1 \\
\hline & $28-37$ & 53 & 12.9 \\
\hline & $38-47$ & 22 & 5.4 \\
\hline & $48-57$ & 12 & 2.9 \\
\hline & $58-67$ & 20 & 4.9 \\
\hline & $68-77$ & 4 & 0.9 \\
\hline Age (years) mean (SD) & $3.3(2.1)$ & & \\
\hline \multirow[t]{2}{*}{ Sex } & Male & 156 & 38.2 \\
\hline & Female & 252 & 61.8 \\
\hline \multirow[t]{4}{*}{ Education level^ } & None & 100 & 34.7 \\
\hline & Primary & 108 & 37.5 \\
\hline & Secondary & 64 & 22.2 \\
\hline & Post secondary & 16 & 5.6 \\
\hline \multirow[t]{4}{*}{ Marital status $^{\circledR}$} & Single & 116 & 62.7 \\
\hline & Cohabiting & 21 & 11.4 \\
\hline & Married & 38 & 20.5 \\
\hline & Widowed & 10 & 5.4 \\
\hline \multirow[t]{2}{*}{ Employment status $\boldsymbol{\pi}$} & Not employed & 142 & 76.8 \\
\hline & Employed & 43 & 23.2 \\
\hline \multirow[t]{2}{*}{ Type of residence } & Rural & 192 & 47.1 \\
\hline & Urban & 216 & 52.9 \\
\hline \multirow[t]{2}{*}{ Visitor in this district } & Yes & 56 & 13.7 \\
\hline & No & 352 & 86.3 \\
\hline \multirow[t]{2}{*}{ Utilization of malaria diagnostic tests } & Non-utilizers & 49 & 12.0 \\
\hline & Utilizers & 359 & 88.0 \\
\hline \multirow[t]{2}{*}{${ }^{*}$ Utilizers of malaria diagnostic tests } & Positive & 134 & 37.3 \\
\hline & Negative & 225 & 62.7 \\
\hline \multirow[t]{2}{*}{ Receipt of anti-malarial drugs } & Did not receive & 167 & 40.9 \\
\hline & Received & 241 & 59.1 \\
\hline
\end{tabular}

$\mathrm{N}=408$; ${ }^{*}$ Utilizers = only 359 who tested for malaria. Education level^ = only the 288 respondents aged 5 years and above; Marital status ${ }^{\circledR}$ and Employment status $\pi=$ only the 185 respondents aged 18 years and above. 


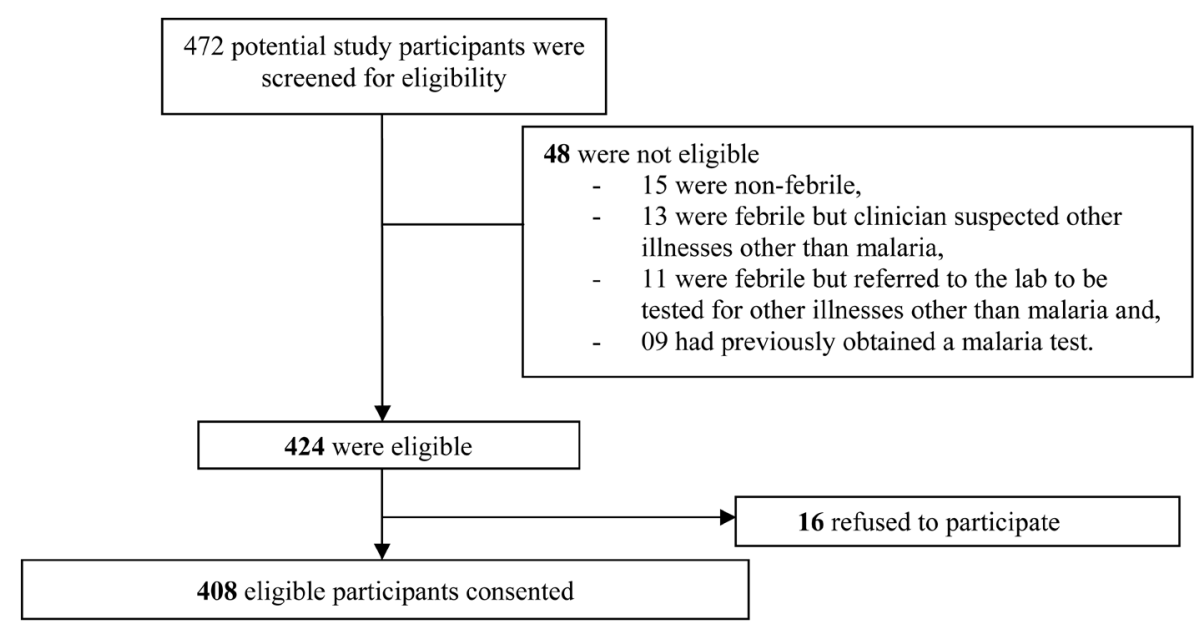

Figure 1. Eligibility screening process of the 472 potential study participants. The final study sample included a total of 408 respondents who consented to participate in the study. Of these, 204 were obtained from Mukono health centre IV, 122 from Kojja health centre IV and 82 from Mukono C.o.U health centre IV.

\subsection{Patients' Factors Associated with Utilization of Malaria Diagnostic Tests}

As shown by the chi square p values in Table 3 and Table 4, there were no statistically significant differences between utilizers and non-utilizers of malaria diagnostic tests in terms of their socio-demographic characteristics except for age, $(\mathrm{p}=0.00)$ history of in-door residual spraying $(\mathrm{p}=0.00)$ and perceived satisfaction with services at outpatient clinics $(\mathrm{p}=0.01)$.

\subsection{Facility-Based Factors Associated with Utilization of Malaria Diagnostic Tests}

As recommended by the Uganda National Malaria Diagnostic Policy, the blood smear microscopy was the only method of malaria diagnosis used in all the three health centre IV facilities [11]. Mukono C.o.U health centre IV was doing better than Mukono health centre IV and Kojja health centre IV in terms of utilities, equipment and supplies as well as skilled laboratory personnel required for microscopic diagnosis of malaria (Figure 2). However, it was observed that much as Mukono C.o.U health centre IV almost had all the resources required for microscopic diagnosis of malaria, this health facility still recorded the least number of prepared blood slides.

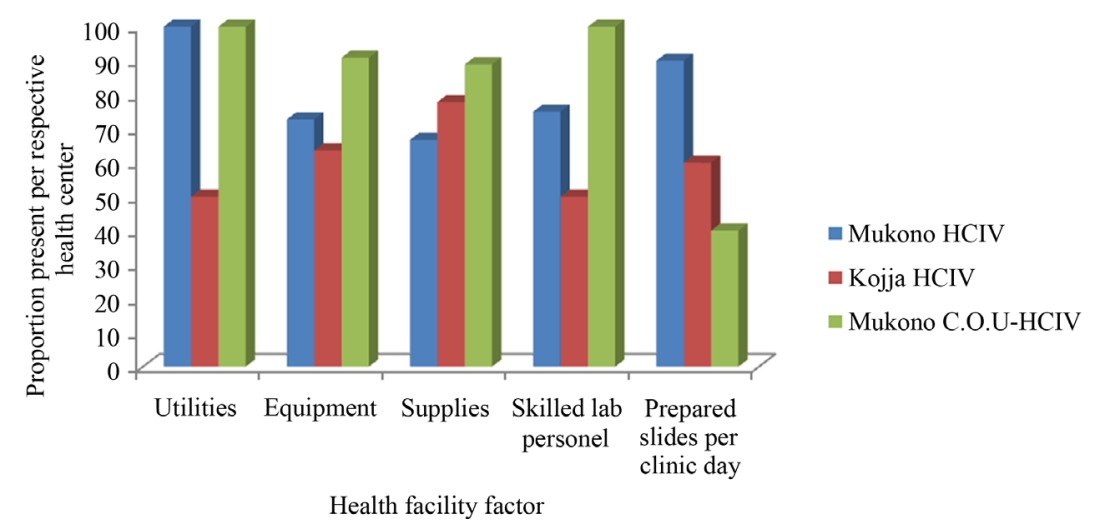

Figure 2. Description of health facility factors associated with utilization of malaria diagnostic tests at out-patient clinics of health centre IV facilities in Mukono district between March and May 2012. Figure 2 shows presence of facility-based factors without which microscopic diagnosis of malaria cannot be performed [6]. Mukono C.o.U health centre IV was doing better than Mukono HC/IV and Kojja HC/IV in terms of utilities, equipment and supplies as well as skilled laboratory personnel required for microscopic diagnosis of malaria. 
Table 3. Patients' factors associated with utilization of malaria diagnostic tests by febrile patients suspected of malaria at out-patient clinics of health centre IV facilities in Mukono district between March and May 2012.

\begin{tabular}{|c|c|c|c|c|}
\hline Variable name & Utilizers n (col \%) (n = 359) & Non-utilizers n (col \%) $(n=49)$ & Total $(\mathrm{N}=408)$ & Pr-value $\left(x^{2}\right)$ \\
\hline \multicolumn{5}{|l|}{ Age-group } \\
\hline $0-4$ & $120(33.4)$ & $0(0.0)$ & 120 & \\
\hline $5-10$ & $56(15.6)$ & $13(26.5)$ & 69 & \\
\hline $11-17$ & $28(7.8)$ & $6(12.2)$ & 34 & \\
\hline $18-27$ & 61 (16.9) & $13(26.5)$ & 74 & \\
\hline $28-37$ & $45(12.5)$ & 8 (16.3) & 53 & \\
\hline $38-47$ & $20(5.6)$ & $2(4.1)$ & 22 & \\
\hline $48-57$ & $11(3.1)$ & $1(2.0)$ & 12 & \\
\hline $58-67$ & $17(4.7)$ & $3(6.1)$ & 20 & \\
\hline $68-77$ & $1(0.3)$ & $3(6.1)$ & 4 & $0.00^{*}$ \\
\hline \multicolumn{5}{|l|}{ Sex } \\
\hline Male & 136 (37.9) & $20(40.8)$ & 156 & \\
\hline Female & $223(62.1)$ & $29(59.2)$ & 252 & 0.69 \\
\hline \multicolumn{5}{|l|}{ Education level$\wedge^{\wedge}$} \\
\hline None & $79(33.1)$ & $21(42.9)$ & 100 & \\
\hline Primary & 94 (39.3) & $14(28.6)$ & 108 & \\
\hline Secondary & $53(22.2)$ & $11(22.4)$ & 64 & \\
\hline Post secondary & $13(5.4)$ & $3(6.1)$ & 16 & 0.49 \\
\hline \multicolumn{5}{|l|}{ Marital status $^{\circledR}$} \\
\hline Single & $92(59.4)$ & $24(80.0)$ & 116 & \\
\hline Cohabiting & 18 (11.6) & $3(10.0)$ & 21 & \\
\hline Married & $36(23.2)$ & $2(6.7)$ & 38 & \\
\hline Widowed & $9(5.8)$ & $1(3.3)$ & 10 & 0.14 \\
\hline \multicolumn{5}{|c|}{ Employment status $\pi$} \\
\hline Not employed & $115(74.2)$ & $27(90.0)$ & 142 & \\
\hline Employed & $40(25.8)$ & $3(10.0)$ & 43 & 0.06 \\
\hline \multicolumn{5}{|l|}{ Type of residence } \\
\hline Rural & $167(46.5)$ & $25(51.0)$ & 192 & \\
\hline Urban & $192(53.5)$ & $24(49.0)$ & 216 & 0.55 \\
\hline \multicolumn{5}{|l|}{ Visitor/tourist } \\
\hline Yes & $51(14.2)$ & $5(10.2)$ & 56 & \\
\hline No & 308 (85.8) & $44(89.8)$ & 352 & 0.45 \\
\hline \multicolumn{5}{|l|}{ Time of arrival } \\
\hline Morning & 68 (18.9) & $0(0.00)$ & 68 & \\
\hline Mid-morning & 225 (62.7) & $28(57.1)$ & 253 & \\
\hline Afternoon & $66(18.4)$ & $21(42.9)$ & 87 & $0.00^{*}$ \\
\hline
\end{tabular}

${ }^{*}$ with font bolded denotes a statistically significant difference between utilizers and non-utilizers at $\mathrm{p}<0.05$; Education level $\wedge=$ only the 288 respondents aged 5 years and above; Marital status ${ }^{\circledR}$ and Employment status $\pi=$ only the 185 respondents aged 18 years and above. 
Table 4. Personal health behaviour and overall perceived satisfaction with services in relation to utilization of malaria diagnostic tests by febrile patients suspected of malaria at outpatient clinics of health centre IV facilities in Mukono district between March and May 2012.

\begin{tabular}{|c|c|c|c|c|}
\hline Variable name & $\begin{array}{c}\text { Utilizers } \\
\text { n }(\mathbf{c o l} \%)(n=359)\end{array}$ & $\begin{array}{c}\text { Non-utilizers } \\
n(\mathrm{col} \%)(n=49)\end{array}$ & $\begin{array}{c}\text { Total } \\
(\mathrm{N}=\mathbf{4 0 8})\end{array}$ & $\begin{array}{c}\text { Pr-value } \\
\left(x^{2}\right)\end{array}$ \\
\hline \multicolumn{5}{|c|}{ Slept under a treated mosquito net in past 14 days } \\
\hline Yes & $292(81.3)$ & $36(73.5)$ & 328 & \\
\hline No & $67(18.7)$ & $13(26.5)$ & 80 & 0.19 \\
\hline \multicolumn{5}{|c|}{ Sprayed inside house in the past 6 months } \\
\hline Yes & $53(14.8)$ & $19(38.8)$ & 72 & \\
\hline No & $306(85.2)$ & $30(61.2)$ & 336 & $0.00^{*}$ \\
\hline \multicolumn{5}{|c|}{ Satisfaction with services } \\
\hline Satisfied & $253(70.5)$ & $26(53.1)$ & 279 & \\
\hline Not satisfied & $106(29.5)$ & $23(46.9)$ & 129 & $0.01^{*}$ \\
\hline
\end{tabular}

${ }^{*}$ with font bolded denotes statistically significant differences between utilizers and non-utilizers at $\mathrm{p}<0.05$.

\subsection{Provider-Related Factors Associated with Utilization of Malaria Diagnostic Tests}

Out of the six health workers purposively selected for interviews, three were clinicians and three were laboratory technicians by training all of whom had reportedly served in their respective cadres for more than two years. There was a "gap" between clinicians and laboratory technicians in terms of their exposure to in-service training and knowledge on malaria treatment guidelines.

\subsubsection{Cadre of Health Workers and Training}

As opposed to clinicians, findings from this study showed that laboratory technicians had attended workshops and on-site practical sessions on the use of malaria diagnostic tests at least twice a year. Clinicians on the other hand, had not had any in-service training in the past two years prior to this survey. As quoted by clinician 1 , " $I$ do not remember having any training on the use of malaria diagnostic tests, it's normally the lab people who go for those workshops and for us, we learn from them".

\subsubsection{Knowledge on Malaria Treatment Guidelines}

Clinicians were more knowledgeable on the current nationally recommended malaria treatment guidelines as opposed to laboratory personnel. Much as laboratory technicians understood the relevance of malaria of being kept up-to-date with the national recommended guidelines for malaria treatment, some of them still perceived it as “none of their business". As quoted by laboratory technician 1, “I don't know about the guidelines, it is only the doctor or the clinician who should be concerned about treatment guidelines. For me, I do not usually need them because am not going to give patients medicine or inject them. I am only concerned about how to come up with a clear diagnostic test result for malaria and there is nothing more”.

\subsubsection{Basis for Referring Malaria Suspects to the Laboratory}

The practice by most clinicians was to refer all febrile patients to the laboratory for malaria diagnosis on the basis of their presenting symptoms. However, absence of power was the main reason as to why malaria diagnostic tests were at times not done. As quoted by laboratory technician 2, "Power is the main limitation here. If there is no power, I will not perform."

\subsubsection{Prescription Practices for Malaria Suspects}

In some cases, clinicians prescribed anti-malarial drugs for malaria suspects without prior diagnosis for malaria such as those who had a reported history of self medication. As quoted by clinician 3, "If somebody is already taking anti-malarial drugs, you still give anti-malarial drugs. So why bother pricking this person for a test”. 


\subsection{Utilization of Malaria Diagnostic Tests and Receipt of Anti-Malarial Drugs}

Out of 408 respondents, the majority (359, 88\%) utilized malaria diagnostic tests and 241 (59\%) received antimalarial drugs. Out of the 359 respondents who tested for malaria (utilizers), 134 (37.3\%) were diagnosed as positive for malaria while the majority of utilizers (225, 62.7\%) tested negative (Table 2). At bivariable analysis, utilizers were $57 \%$ less likely to receive anti-malarial drugs compared to non-utilizers (Crude OR: 0.43 (0.21, 0.85 ) in the absence of other factors (Table 5). At multivariable analysis, this observed association between utilization of malaria diagnostic tests and receipt of anti-malarial drugs even became stronger in the presence of multiple predictor factors. Malaria suspects who tested for malaria (utilizers) were $75 \%$ less likely to receive anti-malarial drugs than those who were not tested (non-utilizers) after controlling for age, sex and type of residence (Adjusted OR: 0.25, 95\% CI: $0.09,0.66$ ). However, time of arrival at the health facility, history of indoor residual spraying, availability of anti-malarial drugs and satisfaction with services at out-patient clinics remained significantly associated with receipt of anti-malarial drugs in the multivariable model (Table 6). This means that each of these variables was independently associated with the receipt of anti-malarial drugs with or without the presence of other factors in the model. With regard to time of arrival at the health facilities, malaria suspects who arrived at the respective health facilities in the afternoon hours of the day were $75 \%$ less likely to receive anti-malarial drugs compared to those who arrived at the health facilities in the morning hours (OR: 0.25, 95\% CI: $0.09,0.69)$. In terms of their history of indoor residual spraying, malaria suspects who had reportedly not sprayed inside their houses in the past six months prior to the interview date were about 2.3 times more likely to receive anti-malarial drugs than those who had sprayed (OR: 2.26, 95\% CI: 1.03, 4.93). Where anti-malarial drugs were reportedly not available at the respective health facilities, malaria suspects were $97 \%$ less likely to receive them compared to where they were available (OR: $0.03,95 \%$ CI: $0.01,0.06$ ).With regard to satisfaction with services at out-patient clinics, un-satisfied patients were $60 \%$ less likely to receive anti-malarial drugs than satisfied patients (OR: 0.40, 95\% CI: 0.21, 0.75).

Table 5. Bivariable analysis for the association between utilization of malaria diagnostic tests (the lead predictor variable) and receipt of anti-malarial drugs by febrile patients suspected of malaria at outpatient clinics of health centre IV facilities in Mukono district between March and May 2012.

\begin{tabular}{ccccc}
\hline $\begin{array}{c}\text { Utilization of malaria } \\
\text { diagnostic tests) }\end{array}$ & $\begin{array}{c}\text { Did not receive anti-malarial } \\
\text { drugs } \mathbf{n}(\mathbf{\%})(\mathbf{n}=\mathbf{1 6 7})\end{array}$ & $\begin{array}{c}\text { Received anti-malarial } \\
\text { drugs } \mathbf{n}(\mathbf{\%})(\mathbf{n}=\mathbf{2 4 1})\end{array}$ & $\begin{array}{c}\text { Total } \mathbf{n}(\%) \\
\mathbf{( N = 4 0 8 )}\end{array}$ & $\begin{array}{c}\text { Crude OR } \\
\mathbf{( 9 5 \%} \mathbf{~ C I})\end{array}$ \\
\hline Non-utilizers (1) & $12(7.2)$ & $37(15.3)$ & $49(12.0)$ & \\
Utilizers & $155(92.8)$ & $204(84.7)$ & $359(88.0)$ & $0.43(0.21,0.85)$ \\
\hline
\end{tabular}

Table 6. Adjusted analysis for the association between utilization of malaria diagnostic tests and receipt of anti-malarial drugs by febrile patients suspected of malaria at outpatient clinics of health centre IV facilities in $\mathrm{Mu}-$ kono district between March and May 2012.

\begin{tabular}{|c|c|c|c|c|}
\hline \multirow{2}{*}{$\begin{array}{c}\text { Variable Name } \\
\text { Utilization of malaria diagnostic tests }\end{array}$} & \multicolumn{2}{|c|}{ Crude Analysis } & \multicolumn{2}{|c|}{ Adjusted Analysis } \\
\hline & $\mathbf{O R}^{\mathrm{c}}$ & $95 \% \mathrm{CI}$ & $\mathbf{O R}^{\mathrm{a}}$ & $95 \%$ CI \\
\hline Non-utilizers & 1 & Reference & 1 & Reference \\
\hline Utilizers & 0.43 & $0.21,0.85^{*}$ & 0.25 & $0.09,0.66^{*}$ \\
\hline Age & 1.01 & $0.99,1.02$ & 1.01 & $0.99,1.03$ \\
\hline \multicolumn{5}{|l|}{ Sex } \\
\hline Male & 1 & Reference & 1 & Reference \\
\hline Female & 0.65 & $0.43,0.99^{*}$ & 0.54 & $0.29,1.01$ \\
\hline
\end{tabular}




\section{Continued}

\section{Type of residence}

Rural

Urban

Time of arrival at the health centre

$$
\text { Morning }
$$

Mid-morning

Afternoon

Sprayed inside house

Yes

No

Satisfaction with services

$$
\begin{gathered}
\text { Satisfied } \\
\text { Not satisfied }
\end{gathered}
$$

Availability of anti-malarial drugs

At this health centre
Outside this health centre

1

0.25

Reference
0.16, 0.39*

1

0.58

\section{1}

1.37

0.25

$0.65,2.35$

Reference

$1.49,4.30^{*}$

Reference

0.23, $0.55^{*}$
Reference

0.32, 1.05

$\mathrm{OR}^{\mathrm{c}}$ and $\mathrm{OR}^{\mathrm{a}}$ means crude and adjusted odds ratios respectively; " with font bolded denotes a statistically significant association at $\mathrm{p}<$ 0.05 at bivariable and multivariable analysis.

\section{Discussion}

\subsection{Utilization of Malaria Diagnostic Tests and Receipt of Anti-Malarial Drugs}

The key finding of this study showed a negative association between utilization of malaria diagnostic tests and receipt of anti-malarial drugs among febrile patients suspected of malaria. In the adjusted analysis, utilizers were $75 \%$ less likely to receive anti-malarial drugs as opposed to non-utilizers after controlling for age, sex and residence. This means that increasing utilization of malaria diagnostic tests among febrile patients suspected of malaria can reduce the problem of over-prescription of anti-malarial drugs by $75 \%$ among those tested for malaria since anti-malarial drugs would be received by only those with a parasitologically-confirmed diagnosis for malaria. This gives an opportunity for febrile patients without a parasitologically-confirmed diagnosis of malaria to be examined for other causes of illness. This finding is consistent with findings from other studies in which the relevance of malaria diagnosis in the receipt of anti-malarial drugs has been demonstrated. For example, in a clinical trial done in Zanzibar to assess the influence of Rapid Diagnostic Test (RDT)-aided malaria diagnosis on drug prescriptions in primary health care settings, RDT was associated with lower prescription rates of antimalarial treatment (OR: 0.04, 95\% CI: 0.03, 0.05) [12].

\subsection{Socio-Demographic Characteristics of Study Participants}

In this study, the majority of respondents were female below five years of age. This finding is consistent with a cross-sectional survey conducted in 2008 on prevalence of malaria in Mukono district in which the majority of respondents (77.5\%) were female and $67 \%$ out of 2897 households surveyed reported at least a child with malaria two weeks prior to the survey date, thus indicating a high malaria prevalence among under fives in the district [2].

\subsection{Patient Factors Associated with Utilization of Malaria Diagnostic Tests}

In this study, utilizers did not differ statistically from non-utilizers in terms of their socio-demographic characte- 
ristics except age, history of indoor spraying and time of arrival at the respective health centres. The findings of this study show that a majority of febrile patients utilized malaria diagnostic tests. This can be possibly explained from the clinician's self reports in which they cited that they would only refer a patient to the laboratory on the basis of their presenting symptoms and specifically for a malaria test if they were febrile. This was evidenced in a study done in Tanzania in which health workers' prescription patterns for anti-malarial drugs decreased from $89 \%$ to $38 \%$ after the policy changed to treatment based on parasitologically-confirmed diagnosis [13].

\subsection{Facility-Based Factors Associated with Utilization of Malaria Diagnostic Tests}

On average, all health centre IV facilities in Mukono district were equipped with the necessary resources needed for microscopic diagnosis of malaria, although largely constrained by frequent power cut-offs, a situation which demotivated the health workers as further evidenced by a case study in Tanzania in which supply-related factors such as supplies and equipment were drivers of staff motivation [6] [14]. Findings from this study show that Mukono C.o.U health centre IV was doing better than Mukono health centre IV and Kojja health centre IV in terms of utilities, equipment and supplies as well as skilled laboratory personnel required for microscopic diagnosis of malaria. However, Mukono C.o.U health centre IV incidentally had the least average number of prepared blood slides per clinic day as opposed to Mukono health centre IV and Kojja health centre IV. This can be possibly explained by the fact that at Mukono C.o.U health centre IV, an additional fee was attached to a malaria test unlike Mukono health centre IV and Kojja health centre IV where no additional costs for conducting a malaria test were incurred by the patient.

\subsection{Provider-Related Factors Associated with Utilization of Malaria Diagnostic Tests}

Contrary to laboratory personnel, all clinicians had not obtained any in-service training on the use of malaria diagnostic tests irrespective of how long they had served. This is because most of the in-service trainings held on the use of malaria diagnostic tests in the district were often exclusively attended by laboratory personnel. This finding is consistent with an exploratory study done in South Africa in which $60 \%$ of the nurses did not receive any external training on conducting and interpreting RDTs [15]. In addition, a knowledge gap on malaria treatment guidelines existed amongst laboratory personnel. This may be attributed to their misperception that malaria treatment guidelines do not concern them but exclusively apply to clinicians and doctors. This gap may be further explained by the fact that in-service trainings in the past have been targeting specific cadre of health personnel instead of encompassing all cadres. For example, laboratory personnel reported having attended several workshops and practical sessions on the use of malaria diagnostic tests in the past exclusive of other cadres.

\section{Conclusion}

Utilization of malaria diagnostic tests was high (88\%) and about $60 \%$ of febrile patients received anti-malarial drugs irrespective of whether they had obtained a malaria test or not. Utilizers were $75 \%$ less likely to receive anti-malarial drugs as opposed to non-utilizers after controlling for age, sex and residence. This implies that increasing utilization of malaria diagnostic tests among febrile patients suspected of malaria can reduce the problem of over-prescription of anti-malarial drugs by $75 \%$ among those tested for malaria since anti-malarial drugs would be received by or directed to only those with a parasitologically-confirmed diagnosis of malaria, thereby giving a chance to those without a parasitologically-confirmed diagnosis of malaria to be examined for other causes of illness. However, microscopic diagnosis of malaria was largely constrained by absence of electric power and laboratory personnel especially at night.

\section{Recommendations and Policy Implications}

To be able to overcome the problem of over-prescription of anti-malarial drugs, efforts to boost utilization of malaria diagnostic tests should be one of the key government strategies. A policy that ensures a consistent electric power supply in all public health laboratories should be put in place to make microscopic diagnosis of malaria possible throughout the day. Training of health workers at the district should encompass all cadres of health workers. For instance, if a workshop on malaria treatment guidelines is organized at the district, it should not only target clinicians, laboratory personnel ought to be part. Similarly, if it’s a workshop held on malaria diag- 
nostic tests, it should not be exclusive for laboratory personnel, clinicians too should participate.

Management of health facilities should consider establishing work-shifts for laboratory personnel to enhance utilization of malaria diagnostic tests especially at night. However, more research is needed to further explore the influence of a malaria test result on the receipt of anti-malarial drugs among febrile patients.

\section{Author's Contributions}

NR conceptualized the initial research idea, developed it into a proposal, collected data, analyzed, interpreted and discussed the research findings while AM provided overall direction for research. He also reviewed and added intellectual content to the manuscript. AK and HB respectively closely supervised, corrected and guided NR through the different stages of the design, implementation, synthesis of findings and report writing. They additionally reviewed and helped to streamline the final manuscript. All authors read and approved the submission of the final manuscript for publication.

\section{Acknowledgements}

I am extremely grateful to the Malaria Clinical and Operational Health services Research (COHRE) training program (Grant No. D43TWO08077-01A1) which sponsored this study with support from Fogarty International Centre (FIC) at the National Institutes of Health (NIH). The contents of this study are solely the responsibility of the authors and do not necessarily reflect the views of FIC or NIH. I would like to appreciate the District Health Officer of Mukono district plus the management of the respective health facilities where this study was conducted for their tireless support rendered through the data collection process. I would also like to extend my gratitude to my Academic mentor, Dr. Aggrey Mukose for every encouragement and guidance rendered during my walk through the Master of Health Services Research programme. Lastly, but not least, I do appreciate my classmates in the 2010/2012 MHSR “cohort” for their continued support throughout the programme.

\section{References}

[1] WHO (2013) World Malaria Report.

[2] Mbonye, A.K., Bygbjerg, I.C. and Magnussen, P. (2008) Prevention and Treatment Practices and Implications for Malaria Control in Mukono District, Uganda. Journal of Biosocial Science, 40, 283-296. http://dx.doi.org/10.1017/S0021932007002398

[3] DHO (2011) Monthly Report for Mukono District Health Office. Ministry of Health, Uganda.

[4] Bbosa, R.S. and Ehlers, V.J (2011) Caregivers’ Home-Based Management of Fever in Uganda. African Journal of Nursing and Midwifery, 13, 46-57.

[5] Mbonye, A.K., Ndyomugyenyi, R., Turinde A., et al. (2010) The Feasibility of Introducing Rapid Diagnostic Tests for Malaria in Drug Shops in Uganda. Malaria Journal, 9, 367.

[6] Chanda, P., Marianela, C.-R. and Masiye, F. (2009) Cost-Effectiveness Analysis of the Available Strategies for Diagnosing Malaria in Outpatient Clinics in Zambia. Cost Effectiveness and Resource Allocation, 7, 5.

[7] Angira, C.H., Otieno, O.A., Muga, R.O. and Abong’o, B.O. (2010) Factors Contributing to Antimalarial Drug Resistance in Rachonyo District, Kenya. East African Journal of Public Health, 7, 11-15.

[8] Lammeck, K., Kalibbala, H.M. and Pamela, T. (2010) Environmental and Social Impact Assessment for Proposed Renovation and Equipping of Health Facilities in Uganda. M.O. Health, Editor, Kampala.

[9] Nabbuye-Sekandi, J., Makumbi, F.E., Kasangaki, A., Kizza, I.B., Tugumisirize, J., Nshimye, E., Mbabali, S. and Peters, D.H. (2011) Patient Satisfaction with Services in Out-Patient Clinics at Mulago Hospital, Uganda. International Journal for Quality in Health Care, 23, 516-523. http://dx.doi.org/10.1093/intqhc/mzr040

[10] Taylor, E. and Renner, M. (2003) Program Development and Evaluation. Analyzing Qualitative Data. University of Wisconsin-Extension Cooperative Extension-Madison Wisconsin.

[11] Malaria Control Programme and M.o. Health (2011) Uganda National Malaria Control Policy: Current Draft of Malaria Treatment Guidelines.

[12] Msellem, M.I., Martensson, A., Rotllant, G., et al. (2009) Influence of Rapid Malaria Diagnostic Tests on Treatment and Health Outcome in Fever Patients, Zanzibar: A Cross-Over Validation Study. PLOS Medicine, 6, Article ID: e1000070. http://dx.doi.org/10.1371/journal.pmed.1000070

[13] Bastiaens, G.J., Schaftenaar, E., Ndaro, A., et al. (2011) Malaria Diagnostic Testing and Treatment Practices in Three Different Plasmodium falciparum Transmission Settings in Tanzania: Before and After a Government Policy Change. 
Malaria Journal, 10, 76.

[14] Mubyazi, G.M., Bloch, P., Byskov, J., et al. (2012) Supply-Related Drivers of Staff Motivation for Providing Intermittent Preventive Treatment of Malaria During Pregnancy in Tanzania: Evidence from Two Rural Districts. Malaria Journal, 11, 48.

[15] Moonasar, D., Goga, A.E., Frean, J., et al. (2007) An Exploratory Study of Factors That Affect the Performance and Usage of Rapid Diagnostic Tests for Malaria in the Limpopo Province, South Africa. Malaria Journal, 6, 74. 
Scientific Research Publishing (SCIRP) is one of the largest Open Access journal publishers. It is currently publishing more than 200 open access, online, peer-reviewed journals covering a wide range of academic disciplines. SCIRP serves the worldwide academic communities and contributes to the progress and application of science with its publication.

Other selected journals from SCIRP are listed as below. Submit your manuscript to us via either submit@scirp.org or Online Submission Portal.
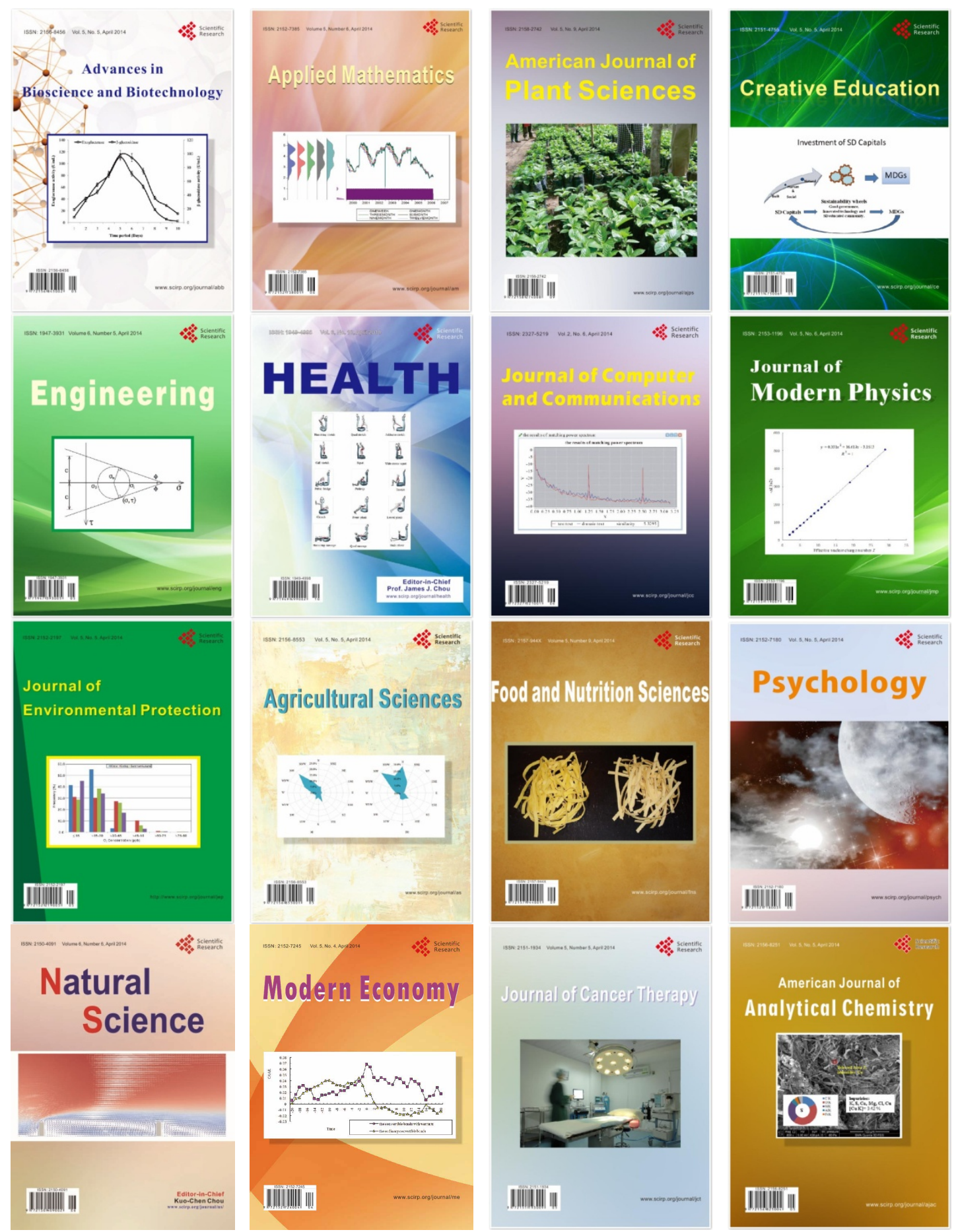